\title{
Enhanced Expression of Podoplanin in Oral Carcinomas in situ and Squamous Cell Carcinomas
}

\author{
Akinori Funayama $^{\mathrm{a}, \mathrm{b}}$ Jun Cheng ${ }^{\mathrm{a}}$ Satoshi Maruyama ${ }^{\mathrm{c}}$ Manabu Yamazaki $^{\mathrm{a}}$

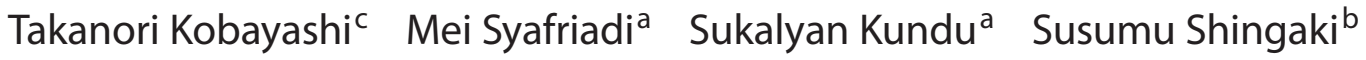 \\ Chikara Saito $^{\text {b }}$ Takashi Saku $^{\text {a,c }}$ \\ Divisions of a Oral Pathology and ${ }^{b}$ Reconstructive Surgery for the Oral and Maxillofacial Region, Department of \\ Tissue Regeneration and Reconstruction, Niigata University Graduate School of Medical and Dental Sciences, and \\ 'Oral Pathology Section, Department of Surgical Pathology, Niigata University Hospital, Niigata, Japan
}

\section{Key Words}

Carcinoma in situ - D2-40 - Epithelial dysplasia - Oral

mucosa $\cdot$ Podoplanin $\cdot$ Squamous cell carcinoma

\begin{abstract}
Objective: Podoplanin, a known lymphatic endothelial cell marker, has been reported to be expressed in various types of cancer. To elucidate the expression of podoplanin in precancerous lesions, we examined the immunohistochemical profiles of podoplanin in oral squamous epithelial lesions. Method: We studied a total of 298 foci of squamous cell carcinoma (SCC), carcinoma in situ (CIS), epithelial dysplasia, and hyperplastic and/or normal epithelial lesions by immunohistochemistry using D2-40. Results: There was no positivity for podoplanin in normal or hyperplastic epithelia, while all of the CIS and SCC foci stained positive. Approximately one third of the mild dysplasia foci (10 of $36 \mathrm{foci}, 28 \%$ ) and $80 \%$ of moderate dysplasia foci (78/98) showed grade 1 positive reactions (positive only in the 1st layer). Grade 2 reactions (up to 4th layer) were seen in 4 of 98 moderate dysplasia foci (4\%), 29 of 74 CIS foci (39\%), and 3 of 30 SCC foci (10\%). Grade 3 reactions (to more than 5th layer) were found
\end{abstract}

in 35 (47\%) CIS foci and 26 (87\%) SCC foci. Conclusions: The relationship between the present histological categorization and podoplanin grade was statistically significant. D240 expression is considered to be related to the severity of oral precancerous lesions.

Copyright $\odot 2011$ S. Karger AG, Basel

\section{Introduction}

In the past half century, the incidence of oral cancer as well as the number of deaths from oral cancer have continued to increase in many countries, including Japan $[1$, 2], while the number of patients with oral precancerous lesions is considered to have also increased in a similar manner. However, precise and objective diagnosis of precancerous lesions is still challenging, as it is dependent on hematoxylin-eosin (HE) findings, because the various types of squamous epithelial lesions that develop in oral mucosa are similar in terms of keratinization tendency. Indeed, the most recent WHO criteria for oral epithelial precursor lesions (2005) [3] remain obscure and essentially the same as those presented in previous versions

\section{KARGER}

Fax +4161306 1234 E-Mail karger@karger.ch www.karger.com
(C) 2011 S. Karger AG, Basel

1015-2008/11/0783-0171\$38.00/0

Accessible online at:

www.karger.com/pat
Prof. Takashi Saku

Division of Oral Pathology, Department of Tissue Regeneration and Reconstruction

Niigata University Graduate School of Medical and Dental Sciences

2-5274 Gakkocho-dori, Chuo-ku, Niigata 951-8514 (Japan)

Tel. +81 25227 2832, E-Mail tsaku@ dent.niigata-u.ac.jp 
$(1997,1971)[4,5]$. This is due to the lack of established scientific tools for their differential diagnosis. However, the difficulties associated with making objective diagnoses of oral precancerous lesions based solely on HEstained sections are much better understood, and a number of studies have been performed to examine objective aids for such diagnoses that are applicable in daily clinical practice. For example, recent immunohistochemical studies have reported the significance of recognition of cellular differentiation (keratins) [6, 7], cell adhesion (Ecadherin and integrins) $[8,9]$, the intraepithelial extracellular matrix (perlecan) [10], and their lyases (MMP-7) [11].

Recently, D2-40 [12], a newly reported monoclonal antibody that recognizes human podoplanin [13], was introduced as an excellent immunohistochemical marker of lymphatic endothelial cells in investigations of neoplasms of lymphatic origin [14], and lymphatic architectures within neoplastic [15] or inflammatory [16] stromata. In a number of studies, the D2-40 antigen unexpectedly became immunolocalized in tumor cells, including those of squamous cell carcinomas (SCCs) in different organs [17-20]. In addition, several reports have described some of the relationships between the expression level of podoplanin and clinical outcome, including lymph node metastases $[18,19,21,22]$. However, there has been no detailed investigation of the characteristics of podoplanin expression in oral precancerous lesions, especially carcinoma in situ (CIS), though it would be of great interest to determine such expression details based on the lines of evidence mentioned above.

In the present study, we examined the immunohistochemical profile of podoplanin in oral precancerous lesions classified using our newly introduced method for recognizing CIS to determine how podoplanin expression is related to the degree of malignancy.

\section{Materials and Methods}

\section{Materials}

A total of 169 surgically removed oral mucosa samples from patients diagnosed with invasive SCC or CIS as well as epulides were selected from the surgical pathology files of the Division of Oral Pathology, Niigata University Graduate School of Medical and Dental Sciences. The surgical specimens simultaneously contained regions of epithelial dysplasia and/or normal epithelium in addition to the main SCC/CIS foci. The histopathological diagnoses were made based on our own criteria utilizing immunohistochemistry for identifying the cell proliferation center (Ki-67), and the extent of cell differentiation toward prickle cells (keratin 13) or basal cells (keratin 19) [6], as described later. In the 169 speci- mens, a total of 298 foci were selected for investigation, including 30 of SCC, 74 of CIS, 98 of moderate dysplasia, 36 of mild dysplasia, and 60 of normal or hyperplastic epithelia. All specimens were routinely fixed in $10 \%$ formalin and embedded in paraffin using routine methods, then serial $3-\mu \mathrm{m}$ sections were cut from the paraffin blocks. One set of sections was stained with $\mathrm{HE}$ and was used for the reevaluation of histological diagnoses, while other sets were used for immunohistochemical evaluations. The experimental protocol for analyzing surgical material was reviewed and approved by the Ethical Board of the Niigata University Graduate School of Medical and Dental Sciences (Oral Life Science).

\section{Antibodies}

The mouse monoclonal antibody D2-40 was purchased from Dako (Glostrup, Denmark; $\operatorname{IgG}_{1}$, diluted at 1:100). A mouse monoclonal antibody against human Ki-67 antigen (clone MIB-1, $\mathrm{IgG}_{1}, 1: 100$; Dako) was used to recognize cells in proliferating phases. Mouse monoclonal antibodies against human keratin 19 (K19, clone RCK 108, IgG 2 a 1:50; Dako) and human keratin 13 (K13, clone DE-K13, IgG 1 , diluted 1:200; Dako) were employed to identify the degree of cell differentiation of squamous epithelial cells toward basal and prickle cells, respectively [6]. For double immunofluorescence for podoplanin versus Ki-67, Alexa Fluor ${ }^{\circledR}$ 568 Dye-conjugated goat antibodies against mouse IgG (for podoplanin; Invitrogen, Life Technologies, Carlsbad, Calif., USA) and Alexa Fluor ${ }^{\circledR} 488$ Dye-conjugated goat antibodies against mouse IgG (for Ki-67; Invitrogen) served as secondary antibodies, respectively.

\section{Immunohistochemistry}

The formalin-fixed, paraffin-embedded serial tissue sections were deparaffinized and subjected to standard immunohistochemical techniques using an EnVision+/HRP system (Dako). After deparaffinization and hydration, sections were rinsed in $0.01 \mathrm{~mol} / \mathrm{l}$ phosphate-buffered saline (PBS, $\mathrm{pH} 7.4$ ). To restore the antigenicity of podoplanin, $\mathrm{Ki}-67$, and $\mathrm{K} 13$, the sections were autoclaved in $0.01 \mathrm{~mol} / \mathrm{l}$ citrate buffer ( $\mathrm{pH} 6.0$ ) at $121^{\circ} \mathrm{C}$ for $10 \mathrm{~min}$ and then left standing for $40 \mathrm{~min}$ to allow reduction to room temperature. To stain for K19, the sections were treated with $0.2 \%$ (w/v) trypsin (type II; Sigma, St. Louis, Mo., USA) in $0.05 \mathrm{~mol} / \mathrm{l}$ Tris- $\mathrm{HCl}$ (pH 7.6) containing $0.1 \% \mathrm{CaCl}_{2}$ at $37^{\circ} \mathrm{C}$ for $30 \mathrm{~min}$ to restore the antigen sites. Following that pretreatment, the sections were rinsed again in PBS and treated with $0.3 \%$ hydrogen peroxide in methanol for $30 \mathrm{~min}$ at room temperature to block endogenous peroxidase activities. After rinsing in PBS containing 0.5\% milk protein (Morinaga Milk Industry, Tokyo, Japan) and $0.05 \%$ Triton X-100 (T-PBS), they were then incubated with $5 \%$ milk protein in T-PBS for $1 \mathrm{~h}$ at room temperature to block non-specific protein binding sites. After incubation with the primary antibodies overnight at $4^{\circ} \mathrm{C}$, the sections were rinsed in T-PBS and treated with polymer-immune complexes (EnVision+peroxidase, rabbit/mouse; Dako) for $1 \mathrm{~h}$ at room temperature. Next, after rinsing with T-PBS, peroxidase reaction products were visualized by incubation with $0.02 \%$ 3,3'-diaminobenzimine (Dohjindo Laboratories, Kumamoto, Japan) in $0.05 \mathrm{~mol} / \mathrm{l}$ Tris- $\mathrm{HCl}$ ( $\mathrm{pH} 7.6$ ) containing $0.005 \%$ hydrogen peroxide. Finally, the sections were counterstained with hematoxylin. For control experiments, the primary antibodies were replaced with pre-immune mouse IgG (Dako). For double-immunofluorescence studies for podoplanin and $\mathrm{Ki}-67$, the anti-Ki-67 antibodies were applied after indirect 
immunofluorescence signals for podoplanin were stabilized by washing with $0.05 \mathrm{~mol} / \mathrm{l}$ glycine- $\mathrm{HCl}(\mathrm{pH} 2.2)$. For single immunofluorescence for podoplanin, nuclear counterstaining was performed by mounting sections with ProLong ${ }^{\circledR}$ Gold Antifade Reagent with DAPI (4',6-diamidino-2-phenylindole; Invitrogen).

\section{Histopathological Diagnostic Criteria for Oral Precancerous} Lesions

Following HE staining and immunohistochemical examinations for K13, K19, and Ki-67, performed as described elsewhere [6], all of the focus samples were classified as (i) normal or hyperplastic epithelia, (ii) epithelial dysplasia, mild and moderate, (iii) CIS, or (iv) SCC. We did not use the category of severe dysplasia because we considered that there was no objective distinction between the so-called severe dysplasia and CIS [6]. Normal/hyperplastic epithelia were defined by the distinct alignment of basal cells as well as by keratinocytic differentiation up to the surface, both of which indicated opposite directions of differentiation from each other (fig. 1a), from the 2 nd basal layer [6]. Ki-67-immunopositive cells were sporadically detected only in the 2 nd basal (parabasal) layer (fig. 1b), while K19 was linearly positive in the 1st layer of basal cells and K13 was positive in the 3rd basal or upper layers (data not shown) [6].

A diagnosis of moderate (true) epithelial dysplasia was made when there was a definite proliferating zone mainly in the lower part of the epithelium with a loss of basal cell alignment (palisading), which was considered to show potential malignancy (fig. 1g). Proliferating basaloid cells located in the lower half of epithelial dysplasia were mostly positive for Ki-67 (fig. 1h). This type of epithelial dysplasia characteristically showed a contrast between upper parakeratinized cells and lower basaloid cells (fig. 1g), which we have termed a 'two-phase appearance' $[6,23]$. In such characteristic two-phase dysplasia, there was no positive staining for K19 or K13 (data not shown) [6]. Epithelial dysplasia of mild (undefined) degree was diagnosed when the epithelium had no definite proliferating cell zones, but showed a partial loss of basal cell alignment (fig. 1d). Ki-67+ cells were increased in number and mainly located in the 2 nd basal (parabasal) layer, though also occasionally in the 1st basal layer (fig. 1e). Staining intensity for K19 was somewhat enhanced in the 1st basal cell layer, while K13 staining was nearly the same as that in normal/hyperplastic epithelia (data not shown) [6].

A diagnosis of CIS was made for not-yet invasive lesions with the following three characteristic subtypes: basaloid, verrucous, and acanthotic. In this study, we did not utilize a category of severe epithelial dysplasia, which is commonly used, because it was difficult to find definite differences between severe dysplasia and CIS in terms of their histological features and immunohistochemical profiles. Neither K19 nor K13 were definitely positive in CIS (not shown) [6]. The basaloid type was characterized by an epithelial layer occupied with extensively proliferating atypical basaloid cells (fig. 2a), which were mostly Ki-67 positive (fig. 2b). This type corresponded to conventional severe dysplasia or CIS according to the WHO standard [3-5]. The verrucous type (fig. 2d) consisted of round-shaped rete ridges, which were composed of unchanged proliferating basaloid cells expanding to the 3rd or 4th cell layers, with dyskeratotic foci in the center. These proliferating basaloid cells did not show basal cell alignment in the 1st layer and most were Ki-67 positive (fig. 2e). The acanthotic type (fig. $2 \mathrm{~g}$ ) was the most differentiated type of CIS, because it contained fewer basaloid cells, while definite prickle cells with a gradual keratinization tendency were seen toward the surface. Ki$67+$ cells were only located in the 1st basal layer with focal expansion to the 2 nd or 3 rd layer (fig. $2 \mathrm{~h}$ ). The other characteristic features of the acanthotic type of CIS included minimal or otherwise overlookable cellular atypia, and steeply indented rete ridges (fig. $2 \mathrm{~g}$ ).

According to the definitions described above, 279 foci were selected and categorized as 41 areas of normal/hyperplastic epithelia, 36 foci of mild dysplasia, 98 foci of moderate dysplasia, 74 foci of CIS, and 30 foci of invasive SCC. Regarding the CIS subtypes, we were able to distinguish 19 basaloid-, 32 verrucous-, and 23 acanthotic-type foci.

Immunohistochemical Evaluation for Podoplanin Expression

Foci of SCC, CIS, moderate and mild dysplasia, and normal/ hyperplastic epithelia, selected based on the above-noted criteria, were evaluated by extension and intensity of the immunohistochemical reaction to the D2-40 antibody. D2-40-positive foci were graded into the following three categories: grade 1, positive reaction in the cell border of mucosal epithelial cells limited to only the 1st basal layer; grade 2, positive in the 1st basal and upper cell layers, but lower than that in the 4th layer; grade 3: positive from the 1st basal to the 5th layer and beyond.

\section{Statistical Analysis}

The frequencies of positive findings in D2-40 immunohistochemistry among the selected foci were compared between each diagnostic category used for oral mucosal lesions. The statistical significance of differences was determined using a $\chi^{2}$ test for independence with Statcel 2, an Excel 2003 add-in (Seiun Inc. OmsPublishing, Tokorozawa, Japan).

\section{Results}

There were characteristic and differential immunohistochemical patterns shown for podoplanin associated with each diagnostic criterion. Table 1 summarizes the immunopositive findings for podoplanin among the studied foci ranging from normal/hyperplastic epithelia to epithelial dysplasia, CIS, and SCC. In the following, immunohistochemical results are separately described for each lesion group.

\section{Normal/Hyperplastic Epithelia}

No podoplanin immunopositivity was detectable in any of the layers of normal epithelia examined (fig. 1a, c). However, lymphatic vessels with open lumina in the lamina propria were clearly demonstrated by positive staining of lymphatic endothelial cells. Obvious positive staining for podoplanin was not found in hyperplastic epithelia covering epulides (fig. 1d, f), even though Ki-67+ cells increased in number in the 2 nd basal or upper layers with no obvious positive cells in the 1st basal layer (fig. 1e). 

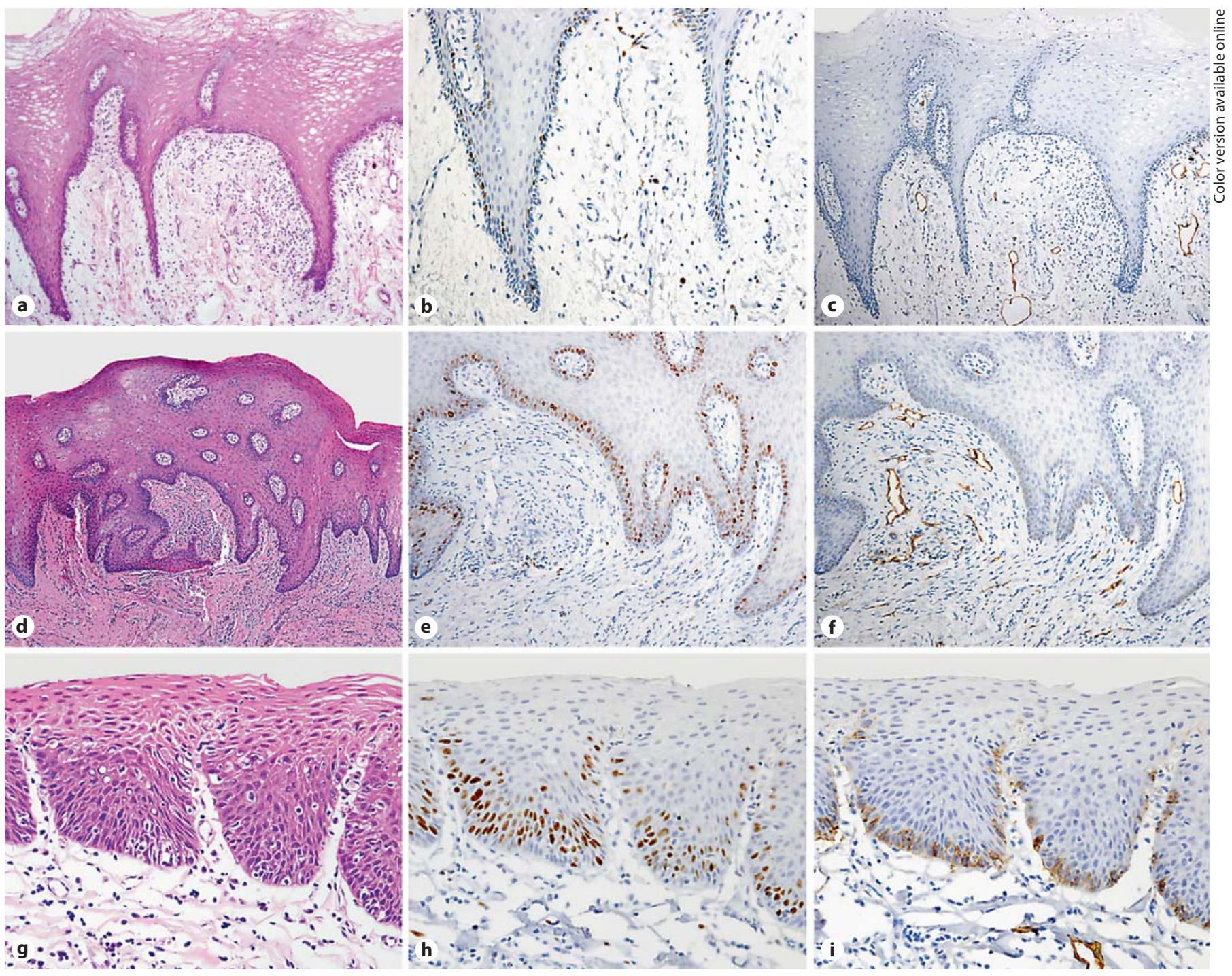

Fig. 1. Normal and dysplastic epithelia of the oral mucosa. a, d, $\mathbf{g ~ H E}$. Immunoperoxidase stain for $\mathrm{Ki}-67$ (b, e, h) and podoplanin (D2-40; c, f, i) and hematoxylin counterstain. a-c Normal epithelia. d-f Reactive hyperplasia. $\mathbf{g}-\mathbf{i}$ Moderate (true) epithelial dysplasia. a, c, $\mathbf{d} \times 100$. b, e, $\mathbf{f} \times 200$. $\mathbf{g}-\mathbf{i} \times 250$. In normal epithelia (a), no podoplanin immunopositivity was detected in any layer (c), while Ki-67-immunopositive cells were sporadically detected only in the 2 nd basal (parabasal) layer (b). In hyperplastic epi-

\section{Epithelial Dysplasia}

The majority of moderate (true) dysplasia foci ( 78 of 98 , 79.6\%) showed immunopositivity for podoplanin in the 1 st basal cell layer (fig. 1i, 3b). This pattern of staining and intensity was evaluated as grade 1 (table 1). In only 4 foci (4.1\%), podoplanin+ cells were stratified focally, though they did not reach more than four cell layers, and were evaluated as grade 2 . Such podoplanin + areas were identi- thelia reactively seen in the covering mucosa of epulides (d), there was no positive staining for podoplanin (f), even though Ki-67+ cells were stratified from the 2 nd basal layer (e). Of note: in the 1st basal cell layer Ki-67+ cells were absent. In moderate epithelial dysplasia with a characteristic two-phase appearance (g), Ki-67+ cells occupied the lower half of the epithelium (h), while podoplanin immunopositivity was sporadically detected in the 1st basal cell layer, especially at the cell borders (i). cal to those in stratified Ki-67+ cells (fig. 1h), though individual podoplanin+ cells were not always positive for Ki-67 (fig. 4a, b). In mild (undefined) dysplasia, one third of the foci $(10 / 36,27.8 \%)$ showed weakly positive staining in the 1st basal cell layer, but the other two thirds did not (fig. 3a). Podoplanin+ lymphatics were observed in the lamina propria similar to those of normal/hyperplastic and mild dysplastic epithelia (fig. 3b). Since podoplanin+ 

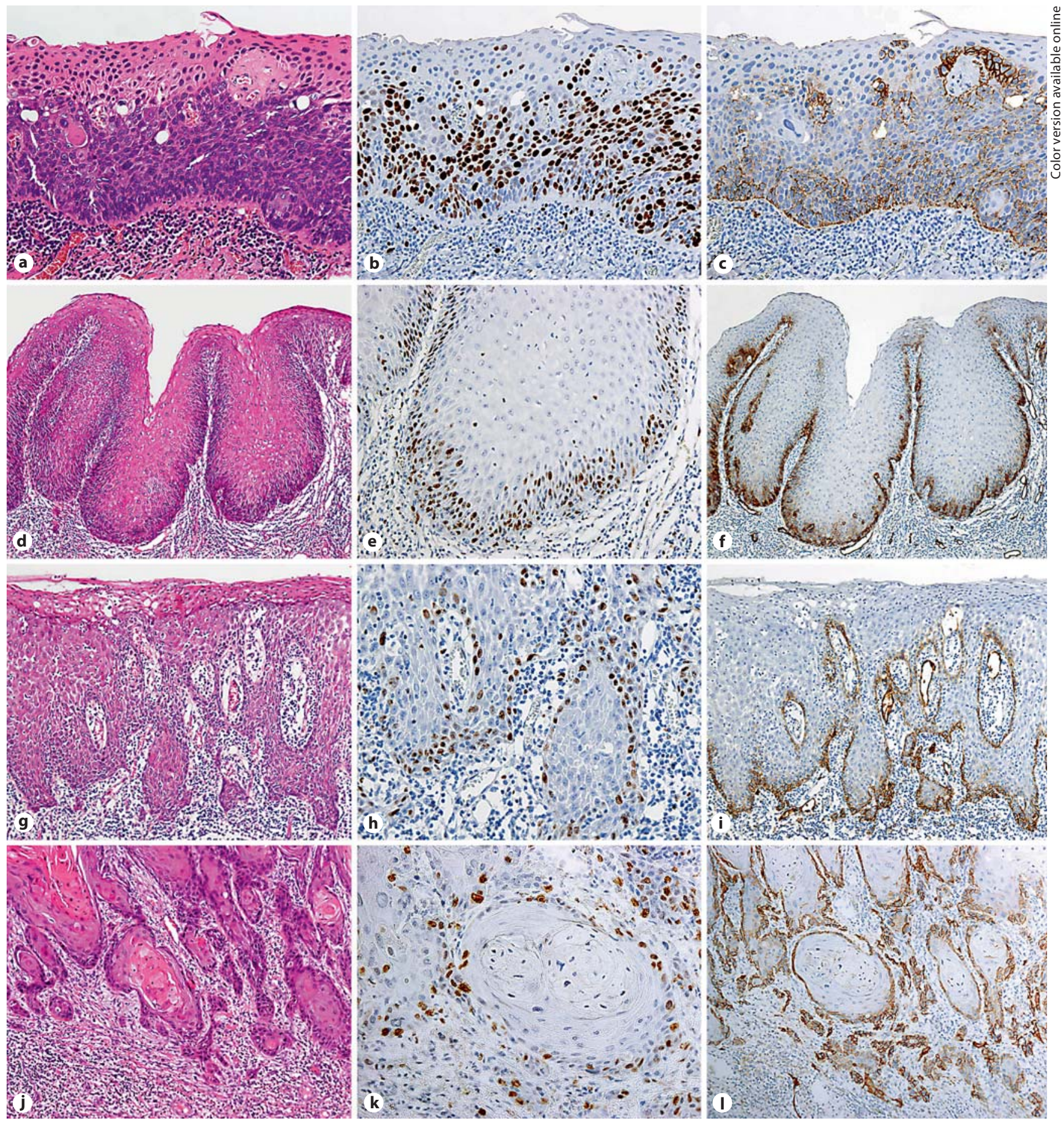

Fig. 2. CIS and SCC of the oral mucosa. $\mathbf{a}, \mathbf{d}, \mathbf{g}, \mathbf{j}$ HE. Immunoperoxidase stain for $\mathrm{Ki}-67(\mathbf{b}, \mathbf{e}, \mathbf{h}, \mathbf{k})$ and podoplanin $(\mathbf{c}, \mathbf{f}, \mathbf{i}, \mathbf{I})$ and hematoxylin counterstain. a-c Basaloid-type CIS. d-f Verrucoustype CIS. g, h, I Acanthotic-type CIS. j-I Well-differentiated SCC. $\mathbf{a - c}, \mathbf{e}, \mathbf{k} \times 200 . \mathbf{d}, \mathbf{f} \times 80 . \mathbf{g}, \mathbf{i} \times 125 . \mathbf{h} \times 250$. j, $\mathbf{I} \times 100$. In basaloid CIS (a), Ki-67+ cells were scattered throughout all the layers (b), while podoplanin+ cells were detected in the larger part of the epithelial layer from the basal to lower prickle cell layers (c). In verrucous CIS (d), Ki-67 (e) and podoplanin (f) were similarly im- munopositive in 3-4 cell layers of the basal zone. In acanthotic CIS $(\mathbf{g})$, which resembled SCC except for invasiveness, Ki-67+ cells were characteristically localized linearly in the 1st basal layer (h), with podoplanin+ cells mostly detected in the 1st basal layer with focal expansion to the 2 nd or 3 rd layers (i). In SCC (j), Ki-67 immunolocalization was basically the same (k) as that for acanthotic CIS (h), while podoplanin+ cells were localized in the periphery of cancer cell nests and most basaloid cells clustered in the invasive front were positive for podoplanin (I). 

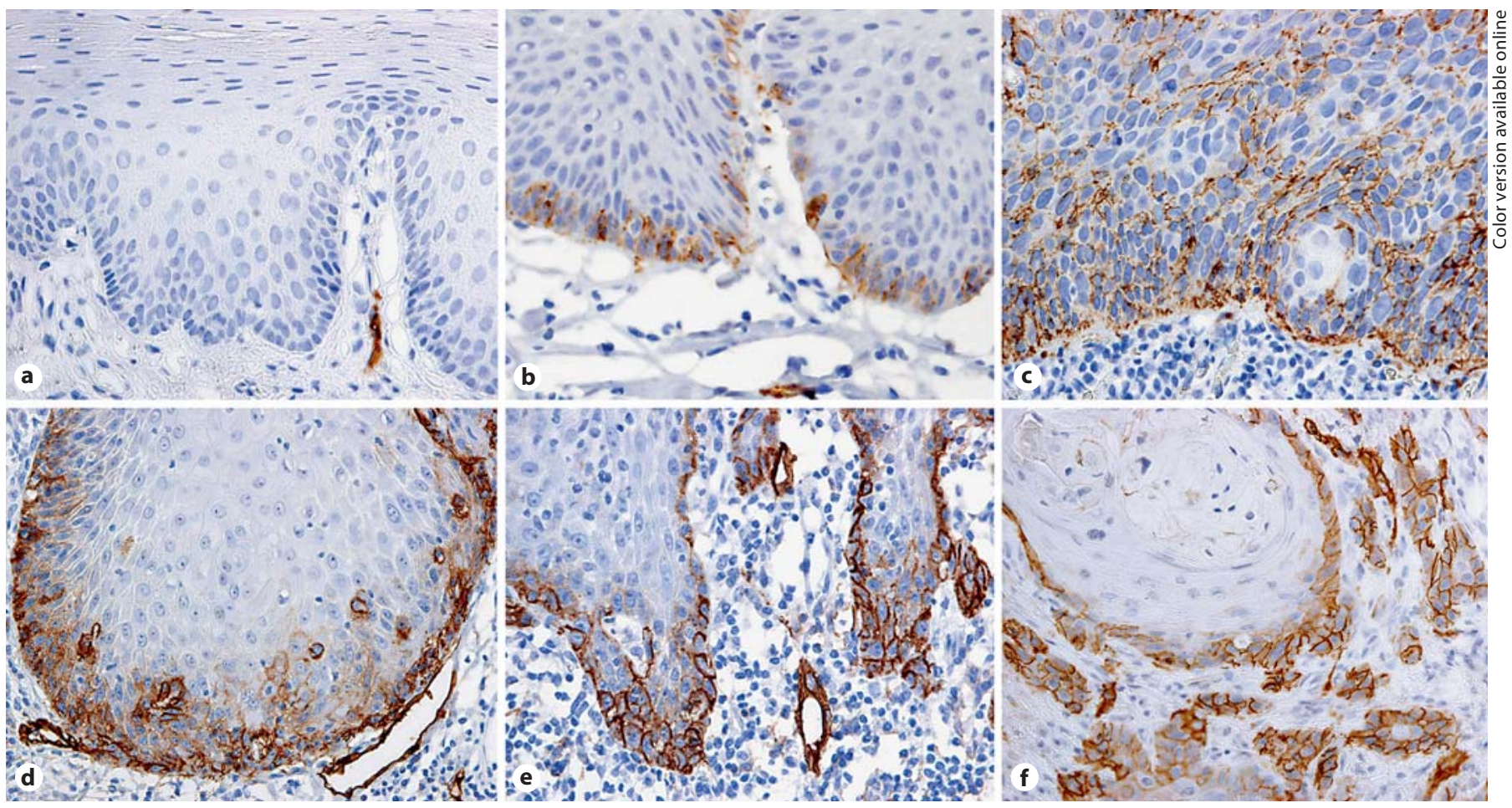

Fig. 3. Epithelial dysplasia, CIS, and SCC of the oral mucosa. a-f Immunoperoxidase stain for podoplanin and hematoxylin counterstain. a Mild dysplasia. b Moderate dysplasia. c Basaloidtype CIS. d Verrucous-type CIS. e Acanthotic-type CIS. f Welldifferentiated SCC. $\mathbf{a}-\mathbf{c}$, e $\times 400$. d $\times 300 ; \mathbf{f} \times 250$. Higher magnifications of the panels in figures 1 and 2. In mild (undefined) dysplasia (and normal and hyperplastic epithelia), podoplanin was not detectable in any epithelial layers, whereas lymphatic vessels were clearly discernible in the lamina propria (a). In moderate (true) dysplasia, podoplanin+ cells were detected in the 1st basal layer (b). In basaloid CIS, podoplanin+ cells were detected in a wide range of epithelial layers from the basal layers to the surface (c). In verrucous CIS, podoplanin was immunolocalized in the 2nd to 3rd basal layers. Lymphatic vessels were closely attached to CIS nests (d). In acanthotic CIS, podoplanin+ cells were rather limited to the 1st basal layer, with focal layering in the peak portion of the rete processes. Small lymphatic vessels were located near each other (e). In SCC, podoplanin+ cells were localized in the periphery of carcinoma cell nests. Most of the basaloid cells consisted of small carcinoma cell strands in the invading front and were positive for podoplanin (f). lymphatics were only located in the connective tissue zone, they were clearly distinguishable from podoplanin+ epithelial cells.

\section{CIS}

Podoplanin was strongly demonstrated in all of the CIS foci, though there were various levels of expression. In CIS, immunolocalization for podoplanin was characteristically enhanced at the cell border and had multiple layers in the basal zone, which was in contrast to epithelial dysplasia. Such continuous podoplanin staining extending to more than five cell layers was evaluated as grade 3 and found more frequently in the 74 CIS foci ( $\mathrm{n}=$ $35,47.3 \%)$ than grade $2(\mathrm{n}=29,39.2 \%)$ and grade $1(\mathrm{n}=$ $10,13.5 \%$; table 1). In basaloid CIS (fig. $2 \mathrm{a}$ ), podoplanin+ cells were extensively detectable in the epithelial layer from the basal layer up to the surface (fig. 2c, 3c), which overlapped with the distribution of Ki-67+ cells (fig. 2b). However, Ki-67+ cells were more widely scattered towards the upper layer (fig. 4c, d). Sixteen of the 19 foci $(84.2 \%)$ were evaluated as grade $2(26.3 \%)$ or grade 3 (57.9\%), while only 3 (15.8\%) showed grade 1 positivity (table 1). In verrucous CIS (fig. $2 \mathrm{~d}$ ), podoplanin (fig. $2 \mathrm{f}$, 3d) and Ki-67 (fig. 2e) were similarly immunolocalized in 3-4 cell layers of the basal zone. Most of the foci (28 of $32,87.5 \%)$ were evaluated as grade $2(43.7 \%)$ or grade 3 (43.8\%; table 1). In acanthotic CIS, which resembled SCC except for invasiveness (fig. 2g), podoplanin+ cells were mainly detected in the 1st basal layer with focal expansion reaching the higher layers in the peak portions of the rete ridges (fig. $2 \mathrm{i}, 3 \mathrm{e}$ ), which was the same as the immunolocalization mode of Ki-67. Grade 2 (43.5\%) and 
Fig. 4. Double immunofluorescence for podoplanin and Ki-67. a, b Moderate dysplasia. c, d CIS. a, c Single immunofluorescence for podoplanin (red) counterstained with DAPI (blue). b, d Double immunofluorescence for podoplanin (red) and $\mathrm{Ki}$ 67 (green). a, b $\times 300$. c, d $\times 400$. In moderate dysplasia, podoplanin was intermittently localized at the cell border of the 1st basal cells (a). Some of the podoplanin+ basal cells were simultaneously positive for Ki-67, while many of them were not positive (b). In CIS in which podoplanin+ cells were stratified (c), there were double-positive cells in the lower zone, while $\mathrm{Ki}-67+$ / podoplanin- cells were scattered in the upper zone (d). Therefore, podoplanin+ cells were located in zones where $\mathrm{Ki}-67+$ cells were condensed, but they were not always in their cell cycle.
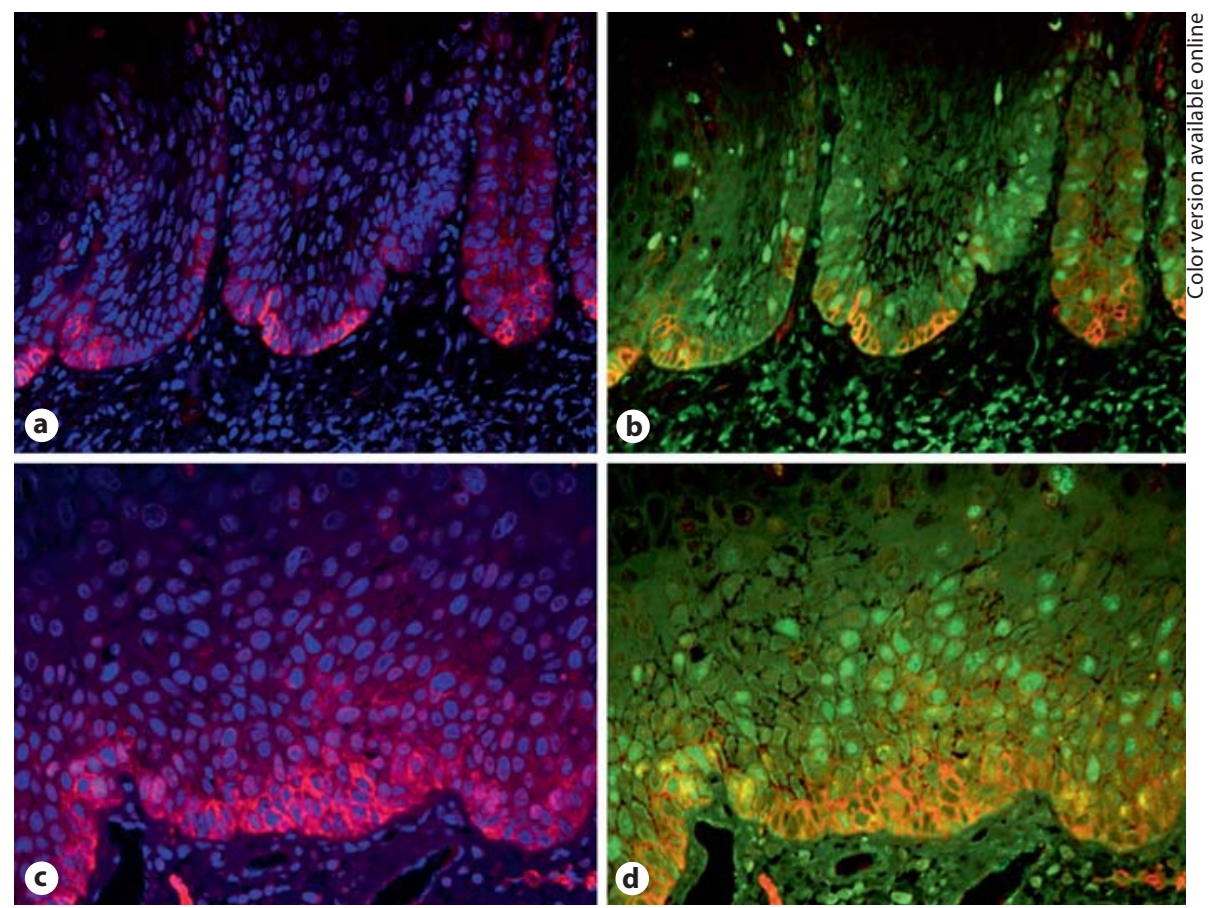

grade 3 (43.5\%) were confirmed in 20 of the 23 foci (total: $87.0 \%)$.

In each type of CIS, podoplanin+ lymphatic vessels were located close to the CIS foci. However, capillary lymphatics were not detectable in the vicinity of the epithelial zone, but rather distant to the lamina propria in nor$\mathrm{mal} /$ hyperplastic epithelia, mild dysplasia (fig. 3a), and moderate dysplasia (fig. 3b). Thus, the distribution mode of lymphatic vessels may also be worthy of note for the differential diagnosis of CIS from not-yet malignant lesions.

\section{SCC}

All of the SCC foci (fig. 2j) showed immunopositivity for podoplanin (table 1). The staining was confined to the peripheral zone of the carcinoma cell nests and mostly continuous, while it was not found in the central area filled with keratinized cells (fig. 2l). In small invading nests or strands, most of the carcinoma cells were positive, especially in the cell border (fig. 3f). Grade 3 staining was found in 26 of the 30 foci $(86.7 \%)$. Grade 2 (3 foci, $10.0 \%$ ) and grade 1 (1 focus, 3.3\%) were evaluated when keratinization was extensive in the foci. Ki-67 immunolocalization was also limited to the periphery of the carcinoma cell nests (fig. 2k), which resembled that of acanthotic CIS (fig. 2h). Within the neoplastic stroma of SCC, there were no apparent lymphatics, while rather dilated and irregular-shaped lymphatics, which were obviously larger than those seen in CIS, were located in the surrounding granulation tissue.

The frequency of the 3 podoplanin positivity grades was compared in the different epithelial lesions using a $\chi^{2}$ test for independence. We found significant correlations among the 3 grades and each histological diagnostic category; normal/hyperplastic epithelia, mild and moderate dysplasia, CIS, and SCC ( $<<0.001)$. Namely, the frequency of grade 1 was significantly different between mild and moderate dysplasia, and between dysplasia and CIS. The frequency rates of grades 2 and 3 were significantly different between moderate dysplasia and CIS, CIS and SCC, and dysplasia and SCC. However, there was no statistically significant difference among the three types of CIS (table 1). Based on these results, we considered that the expression level of podoplanin is altered according to the grade of malignancy, and that podoplanin immunopositivity of grade 2 or 3 indicates malignancy. Our histological criteria for CIS based on the characteristics of Ki$67+$ proliferating cells as well as those of cellular differentiation evaluated by K19/K13 immunohistochemistry findings were also objectively confirmed by the consistency of the lesions with podoplanin immunopositivity of grades 2 and 3. Thus, podoplanin immunohistochemistry findings can be effectively used for distinguishing CIS from epithelial dysplasia. 
Table 1. Podoplanin-immunopositive grading in oral epithelial lesions

\begin{tabular}{|c|c|c|c|c|c|}
\hline \multirow[t]{2}{*}{ Lesions } & \multirow{2}{*}{$\begin{array}{l}\text { Areas } \\
\mathrm{n}\end{array}$} & \multicolumn{4}{|c|}{ Frequency of immunopositive grades, n (\%) } \\
\hline & & grade 1 & grade 2 & grade 3 & total \\
\hline Normal/hyperplasia & 60 & $0(0.0)$ & $0(0.0)$ & $0(0.0)$ & $(0.0)$ \\
\hline Dysplasia & 134 & $88(65.7)$ & $4(3.0)$ & $0(0.0)$ & $(68.7)$ \\
\hline Mild & 36 & $10(27.8)$ & $0(0.0)$ & $0(0.0)$ & $(27.8)$ \\
\hline Moderate & 98 & $78(79.6)$ & $4(4.1)$ & $0(0.0)$ & $(83.7)$ \\
\hline CIS & 74 & $10(13.5)$ & $29(39.2)$ & $35(47.3)$ & $(100.0)$ \\
\hline Basaloid & 19 & $3(15.8)$ & $5(26.3)$ & $11(52.6)$ & $(100.0)$ \\
\hline Verrucous & 32 & $4(12.5)$ & $14(43.7)$ & $14(43.8)$ & $(100.0)$ \\
\hline Acanthotic & 23 & $3(13.0)$ & $10(43.5)$ & $10(43.5)$ & $(100.0)$ \\
\hline SCC & 30 & $1(3.3)$ & $3(10.0)$ & $26(86.7)$ & $(100.0)$ \\
\hline
\end{tabular}

Total areas

298

Grade 1 = Positive reaction only in the 1st basal layer; grade 2 = positive reaction from the basal up to the 4 th layer; grade $3=$ positive reaction from the basal to more than the 5 th layer.

\section{Discussion}

In the present study, we found a distinct correlation between immunohistochemical expression levels of podoplanin and histological grades of oral epithelial lesions, when those were categorized based on their immunohistochemical profiles for K19, K13, and Ki-67 [6]. The immunohistochemical characteristics of podoplanin were evaluated by only referring to the presence or absence of positivity and number of positive cell layers. An advantage of this evaluation method is that no podoplanin immunopositivity is seen in any layer of normal or reactive-hyperplastic epithelia. In contrast, approximately one third of the mild dysplasia foci and most of the moderate dysplasia foci were categorized as grade 1 . A large number of the present CIS foci were evaluated as grade 2 or 3, which was essentially the same tendency for SCC, though there was a statistically significant difference in frequency between CIS and SCC. Regarding podoplanin expression characteristics, CIS and moderate dysplasia could be clearly distinguished, and the three subtypes of CIS were equally evaluated. Thus, our findings indicate that the expression mode of podoplanin in oral mucosal epithelial lesions is related to the grade of malignancy, though it is difficult to indicate directly from the present result what roles podoplanin plays in its positive areas.

The monoclonal antibody D2-40 was originally produced to recognize the M2A antigen, a monomeric 40$\mathrm{kDa}$ surface sialoglycoprotein expressed in testicular germ cell tumors and fetal testes [12]. Later, D2-40 came to be regarded as an excellent immunohistochemical marker of lymphatic endothelial cells [13-15]. In the present study, we found that lymphatics in CIS foci displayed specific characteristics in terms of location and shape, as they were located more closely to CIS foci, and had narrow or closed lumina. Thus, it is suggested that recognition of lymphatic shapes and distribution is another useful aid in the differential diagnosis of CIS from epithelial dysplasia.

In addition to lymphatic endothelial cells in physiological or neoplastic conditions, D2-40 immunohistochemical findings are able to reveal the expression of podoplanin in various kinds of neoplasms, including SCCs of the oral cavity or head and neck [17, 20-22], skin [17], uterine cervix [18, 19], and lung [24]. Among head and neck cancers, higher D2-40 immunoreactivity in SCC cells of the tongue was shown to be associated with more frequent lymph node metastases and is now regarded as a predictor of a poor clinical outcome [21]. However, in cervical SCCs, lower D2-40 immunoreactivity is related to more frequent lymphatic invasion and nodal metastases $[18,19]$. Therefore, whether the prognostic significance of D2-40 immunohistochemical findings is critical remains controversial.

There has been no comprehensive study of the various expression characteristics of podoplanin among the different categories of precancerous lesions of the oral mucosa, though we noted its expression in regenerative or dysplastic epithelial cells of the covering oral mucosa of 
mucous retention cysts [16]. Recently, Kawaguchi et al. [22] reported that podoplanin-positive leukoplakia cases had a tendency to progress to cancer. However, their multivariate analysis based on staining pattern scores did not pay sufficient attention to histological details, or variations in dysplasia and CIS. In the present study, the immunohistochemical expression of podoplanin was related to more malignant or more proliferative lesions when comparatively evaluated using the immunohistochemical profiles of the three additional antigens utilized for the evaluation of cell proliferation and differentiation [6].

With respect to biological functions, podoplanin has not been well characterized. PA2.26, a podoplanin homolog, was shown to be upregulated during epidermal carcinogenesis in a mouse model, though it was absent from non-tumorigenic epidermal keratinocytes, suggesting some important role in tumor progression [25]. More recently, podoplanin was shown to be co-localized with ezrin, a transmembrane protein that links with radixin and moesin (ERM protein) to actin filaments, while it also facilitated re-localization of ezrin in plasma membrane protrusions such as filopodia to reduce the expression of E-cadherin and, consequently, cell-cell adhesiveness [20], and promoted epithelial-mesenchymal transition due to activation of RhoA [26]. Thus, the biological function of podoplanin in tumor progression remains unclear. Nevertheless, it is at least generally accepted that podoplanin is overexpressed in various malignancies $[12,14,17-19,24]$ including oral and premalignant cancers [20-22].
Noticeable in the present results was the multi-layering of podoplanin+ cells (grades 2 and 3) in CIS and SCC, which may indicate that these podoplanin-expressing cells acquire individual cell-migrating activity within their foci. We previously reported that perlecan, a heparan sulfate proteoglycan of the basement membrane, as well as its lyase MMP-7 accumulated more frequently within the intercellular spaces of advanced grades of (true) epithelial dysplasia and CIS $[10,11]$. The extension of perlecan and MMP-7 immunopositivity seems to be similar to that of podoplanin, which may indicate that carcinoma cells are in active communication with the extracellular milieu within the zone circumscribed by the basement membrane. In addition, nuclear translocation of $\beta$-catenin and loss of E-cadherin have been demonstrated in the Ki$67+$ lower-half zones of oral dysplasia and CIS [9]. In the present study, podoplanin+ cells were essentially located in the lower part of CIS or periphery of SCC foci, which were composed of basaloid cells simultaneously positive for Ki-67, but not in the upper part, where carcinoma cells were differentiated towards keratinization. The finding that all basaloid cells forming small-strand- or cord-like foci of SCC were positive for podoplanin suggests that podoplanin+/Ki-67+ CIS cells are ready to migrate.

\section{Acknowledgments}

This work was supported in part by grants-in-aid for scientific research from the Japan Society for the Promotion of Science and from the Ministry of Education, Culture, Sports, Science and Technology, Japan.

\section{References}

-1 Franceschi S, Bodoli E, Herrero R, Muñoz N: Comparison of cancers of the oral cavity and pharynx worldwide: etiological clues. Oral Oncol 2000;36:106-115.

2 Statistic and Information Department, the Minister's Secretariat, Ministry of Health, Labour and Welfare, Japan, Health and Welfare Statistics Association: Vital Statistics of Japan 2006. Tokyo, Yamatosogo Publishing, 2006, pp 270-275.

3 Gale N, Piclch BZ, Sidransky D, El Naggar A, Westra W, Califano J, Johnson N, MacDonald DG: Epithelial precursor lesions; in Barnes L, Evson JW, Reichart P, Sidransky D (eds): Pathology and Genetics of Head and Neck Tumours. World Health Organization Classification of Tumours. Lyon, IARC Press, 2005, pp 177-179.
4 Pindborg JJ, Reichart PA, Smith CJ, van der Waal I: Squamous epithelial dysplasia: Histological typing of cancer and precancer of the oral mucosa; in Pindborg JJ, Reichart PA, Smith CJ, van der Waal I (eds): World Health Organization International Histological Classification of Tumours. Berlin, Springer, 1997, pp 24-26.

5 Wahi PN, Cohen B, Luthra UK, Torloni H: Histological typing of oral and oropharyngeal tumours; in Wahi PN, Cohen B, Luthra UK, Torloni H (eds): International Histological Classification of Tumours. Geneva, World Health Organization, 1971, pp 19, 23.
6 Kobayashi T, Maruyama S, Cheng J, Ida-Yonemochi H, Yagi M, Takagi R, Saku T: Histopathological varieties of oral carcinoma in situ: diagnosis aided by immunohistochemistry dealing with the second basal cell layer as the proliferating center of oral mucosal epithelia. Pathol Int 2010;60:156-166.

-7 Takeda T, Sugihara K, Hirayama Y, Hirano M, Tanuma JI, Semba I: Immunohistological evaluation of Ki-67, p63, CK19 and p53 expression in oral epithelial dysplasias. J Oral Pathol Med 2006;35:369-375.

$>8$ Eriksen JG, Steiniche T, Søgaard H, Overgaard J: Expression of integrins and Ecadherin in squamous cell carcinomas of the head and neck. APMIS 2004;112:560568. 
9 Alvarado CG, Maruyama S, Cheng J, Ida-Yonemochi H, Kobayashi T, Yamazaki M, Takagi R, Saku T: Nuclear translocation of $\beta$ catenin synchronized with loss of E-cadherin in oral epithelial dysplasia with a characteristic two-phase appearance. Histopathology, in press.

-10 Ikarashi T, Ida-Yonemochi H, Ohshiro K, Cheng J, Saku T: Intraepithelial expression of perlecan, a basement membrane-type heparan sulfate proteoglycan reflects dysplastic changes of the oral mucosal epithelium. J Oral Pathol Med 2004;33:87-95.

-11 Tilakaratne WM, Kobayashi T, Ida-Yonemochi H, Swelam W, Yamazaki M, Mikami T, Alvarado CG, Shahidul AM, Maruyama S, Cheng J, Saku T: Matrix metalloproteinase 7 and perlecan in oral epithelial dysplasia and carcinoma in situ: an aid for histopathologic recognition of their cell proliferation centers. J Oral Pathol Med 2009;38: 348-355.

12 Marks A, Sutherland DR, Bailey D, Iglesias J, Law J, Lei M, Yeger H, Banerjee D, Baumal R: Characterization and distribution of an oncofetal antigen (M2A antigen) expressed on testicular germ cell tumours. Br J Cancer 1999;80:569-578

13 Evangelou E, Kyzas PA, Trikalinos TA: Comparison of the diagnostic accuracy of lymphatic endothelium markers: Bayesian approach. Mod Pathol 2005; 18:1490-1497.

14 Breiteneder-Geleff S, Soleiman A, Kowalski H, Horvat R, Amann G, Kriehuber E, Diem K, Weninger W, Tschachler E, Alitalo K, Kerjaschki D: Angiosarcomas express mixed endothelial phenotypes of blood and lymphatic capillaries: podoplanin as a specific marker for lymphatic endothelium. Am J Pathol 1999; $154: 385-394$
15 Saad RS, Kordunsky L, Liu YL, Denning KL, Kandil HA, Silverman JF: Lymphatic microvessel density as prognostic marker in colorectal cancer. Mod Pathol 2006;19:13171323.

16 Kundu S, Cheng J, Maruyama S, Suzuki M, Kawashima H, Saku T: Lymphatic involvement in the histopathogenesis of mucous retention cyst. Pathol Res Pract 2007;203:8997.

17 Schacht V, Dadras SS, Johnson LA, Jackson DG, Hong YK, Detmar M: Up-regulation of the lymphatic marker podoplanin, a mucintype transmembrane glycoprotein, in human squamous cell carcinomas and germ cell tumors. Am J Pathol 2005; 166:913-921.

18 Dumoff KL, Chu C, Xu X, Pasha T, Zhang PJ, Acs G: Low D2-40 immunoreactivity correlates with lymphatic invasion and nodal metastasis in early-stage squamous cell carcinoma of the uterine cervix. Mod Pathol 2005;18:97-104

19 Dumoff KL, Chu CS, Harris EE, Holtz D, Xu X, Zhang PJ, Acs G: Low podoplanin expression in pretreatment biopsy material predicts poor prognosis in advanced-stage squamous cell carcinoma of the uterine cervix treated by primary radiation. Mod Pathol 2006;19: 708-716.
20 Martin-Villar E, Scholl FG, Gamallo C, Yurrita MM, Munoz-Guerra M, Cruces J, Quintanilla M: Characterization of human PA2.26 antigen (T1alpha-2, podoplanin), a small membrane mucin induced in oral squamous cell carcinomas. Int J Cancer 2005;113:899-910.

21 Yuan P, Temam S, El-Naggar A, Zhou X, Liu DD, Lee JJ, Mao L: Overexpression of podoplanin in oral cancer and its association with poor clinical outcome. Cancer 2006;107: 563-569.

-22 Kawaguchi H, El-Naggar AK, Papadimitrakopoulou V, Ren H, Fan YH, Feng L, Lee JJ, Kim E, Hong WK, Lippman SM, Mao L: Podoplanin: a novel marker for oral cancer risk in patients with oral premalignancy. J Clin Oncol 2008;26:354-360.

$>23$ Syafriadi M, Cheng J, Jen KY, Ida-Yonemochi H, Suzuki M, Saku T: Two-phase appearance of oral epithelial dysplasia resulting from focal proliferation of parabasal cells and apoptosis of prickle cells. J Oral Pathol Med 2005; 34:140-149.

-24 Kato Y, Kaneko M, Sata M, Fujita N, Tsuruo T, Osawa M: Enhanced expression of Aggrus (T1alpha/podoplanin), a platelet-aggregation-inducing factor in lung squamous cell carcinoma. Tumor Biol 2005;26:195-200.

25 Gandarillas A, Scholl FG, Benito N, Gamalllo C, Quintanilla M: Induction of PA2.26, a cell-surface antigen expressed by active fibroblasts, in mouse epidermal keratinocytes during carcinogenesis. Mol Carcinog 1997; 20:10-18.

\26 Martin-Villar E, Megías D, Castel S, Yurrita MM, Vilaró S, Quintanilla M: Podoplanin binds ERM proteins to activate RhoA and promote epithelial-mesenchymal transition. J Cell Sci 2006;119:4541-4553. 